\title{
有机氯脱氯转化的铁还原菌与铁氧化物界面的 交互反应
}

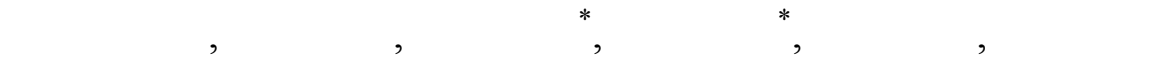 \\ (1) 广东省农业环境综合治理重点实验室, 广东省生态环境与土壤研究所, 广州 510650; \\ (2) 中国科学院广州地球化学研究所, 广州 510640; \\ (3) 华南农业大学资源与环境学院, 广州 510642; \\ (4) 华南理工大学化学与化工学院, 广州 510641; \\ (5) 中国科学院研究生院, 北京 100039 \\ * 联系人, E-mail: cefbli@soil.gd.cn; sgzhou@soil.gd.cn \\ 2008-10-25 收稿, 2009-04-14 接受 \\ 国家自然科学基金资助项目(批准号: 40601043, 40771105)
}

摘要 脱色希瓦氏菌(Shewanella decolorationis, S12)是一株异化铁还原菌, 在厌氧条件下能够以 三价铁 $(\mathrm{Fe}(\mathrm{III}))$ 为末端电子受体, 将其还原成亚铁 $(\mathrm{Fe}(\mathrm{II}))$. 本研究以脱色希瓦氏菌、铁氧化物 $(\alpha-$ $\mathrm{FeOOH}$ )与有机氯(三氯甲烷和五氯酚)三者为基本要素, 构建了一个有机氯脱氯转化的铁还原菌针铁矿界面交互反应体系. 结果表明, S12 对照体系的直接脱氯效果较弱, $\alpha-\mathrm{FeOOH}$ 非生物体系 具有一定的脱氯效果, $\mathrm{S} 12+\alpha-\mathrm{FeOOH}$ 交互反应体系的脱氯转化动力学显著提高. 体系中铁物种 浓度的变化和氧化还原性能的表征结果显示, 铁还原菌 S12 促进界面脱氯转化的主要原因是 S12 能够有效促进吸附态 $\mathrm{Fe}(\mathrm{II})$ 的生成, 并持续提高体系的还原能力. 这些结果将为铁还原菌-铁氧 化物界面可还原性毒害物的脱毒转化研究提供借鉴.

关键词 交互反应 还原脱氯 铁还原菌 针铁矿 三氯甲烷 五氯酚 有机氯(chlorinated organic compounds)是一类重 要的土壤与地下水污染物, 具有难降解性、生物积累 性、致癌致畸性、内分泌干扰性等特性，目前其污染 已经威胁到农产品安全 ${ }^{[1]}$ 与人类健康 ${ }^{[2]}$, 有机氯的迁 移转化已成为国际土壤科学与环境化学的研究热点. 三氯甲烷 ${ }^{[3]}$ (chloroform, CF) 和五氯酚 ${ }^{[4]}$ (pentachlorophenol, PCP)是典型的有机氯污染物. 五氯酚广泛存 在于南方稻田土壤和水体中. 有机氯的毒性主要来 源于其分子结构上的氯原子, 脱除氯原子能显著降 低其生物毒性与环境风险.

厌氧条件下, 还原脱氯是土壤有机氯降解的重 要途径. 研究表明, 水稻土、潮土中有机氯含量明显 低于旱地土壤 [5]. 吸附态与络合态 $\mathrm{Fe}(\mathrm{II})$ 物种被认为
是有机氯脱氯转化的活性物种, 在厌氧环境下通过 电子传递作用促进有机氯的还原脱氮过程 ${ }^{[6,7]}$. 然而, 铁在自然环境中通常以难溶的 $\mathrm{Fe}$ ( III) 氧化物形式存 在. 土壤中 $\mathrm{Fe}$ ( III) 还原溶解生成 Fe( II), 主要是由特 定的异化铁还原微生物驱动的酶促反应. 异化铁还 原细菌(dissimilatory iron-reducing bacteria)能够氧化 葡萄糖、有机酸等电子供体, 并通过呼吸作用将电子 转移至细胞外的铁氧化物表面, 将 $\mathrm{Fe}$ ( III)还原成 $\mathrm{Fe}(\mathrm{II})$, 从中获得能量 ${ }^{[8]}$.

土壤界面生物与非生物交互反应过程是土壤化 学研究的重要热点与难点. 土壤界面有机氯等可还 原性毒害物的脱毒转化过程是一个界面交互反应过 程, 关键活性物种是吸附态 $\mathrm{Fe}(\mathrm{II})$. 铁氧化物发生异 
化还原溶解，并使得有机氯发生化学脱氯反应过程, 实现了铁氧化物异化还原溶解与化学脱氯相结合的 交互反应.

本研究以 $\alpha-\mathrm{FeOOH}($ 针铁矿)、脱色希瓦氏菌 [9] (Shewanella decolorationis, S12)和有机氯(三氯甲烷 和五氯酚), 构建“铁还原菌-针铁矿-有机氯”交互反 应体系. 重点考察这一交互反应体系中有机氯的脱 氯转化效果, 并探讨其交互反应机制.

\section{1 实验与材料}

脱色希瓦氏菌 $\mathrm{S} 12^{[9]}$ 由广东省微生物研究所提供. $\alpha-\mathrm{FeOOH}$ 的制备方法见文献 [10]. S12 $+\alpha-\mathrm{FeOOH}$ 交 互反应体系的成分包括乳酸钠 $10 \mathrm{mmol}$, 三氯甲烷 $0.067 \mathrm{mmol}$ 或五氮酚 $0.076 \mathrm{mmol}, \alpha-\mathrm{FeOOH} 50 \mathrm{mmol}$, 超纯水定容至 $1 \mathrm{~L}, \mathrm{pH} 7.0$. 在 $30^{\circ} \mathrm{C}$ 的 $\mathrm{LB}$ 培养基好氧 培养 $12 \mathrm{~h}$ 的菌体经 $7000 \mathrm{r} / \mathrm{min}$ 离心 $20 \mathrm{~min}$, 无菌超纯 水洗涤重悬浮, 此操作重复 3 次, 最终配成一定浓 度的菌悬液, 终浓度为 $7 \times 10^{8}$ 个细胞 $/ \mathrm{mL}$ 的菌体接 种于反应体系中. 此外, 设计两组对照体系: 非生 物反应体系中不含 $\mathrm{S} 12$ 菌体; 生物反应体系中不含 $\alpha$ $\mathrm{FeOOH}$.

反应体系分装于 $25.2 \mathrm{~mL}$ 的西林瓶中, 用高纯氮 充气 $1 \mathrm{~h}$ 排氧, 然后用橡胶塞压紧, 并立即压铝盖密 封. 样品置于厌氧培养箱中 $(30 \pm 1)^{\circ} \mathrm{C}$ 静置培养. 每隔 一段时间取样, 测定样品中目标物、氯离子和吸附态 $\mathrm{Fe}(\mathrm{II}) / \mathrm{Fe}(\mathrm{III})$ 浓度. 每个样品设 3 个重复, 样品经 $7000 \mathrm{r} / \mathrm{min}$ 离心去除菌体和铁氧化物后, 取上层清液测 定其中氯离子浓度, 采用离子色谱仪(Dionex ICS-90), 配备离子色谱柱(IonPac AS14A $4 \times 250 \mathrm{~mm}$ ), 流动相 为 $\mathrm{Na}_{2} \mathrm{CO}_{3} / \mathrm{NaHCO}_{3}$ 缓冲溶液, 流速为 $1.0 \mathrm{~mL} / \mathrm{min}$. 目标物和 $\mathrm{Fe}(\mathrm{II}) / \mathrm{Fe}(\mathrm{III})$ 浓度的测试方法见参考文献 [7,10].

采用循环伏安方法 $(\mathrm{CV})$ 与传统的三电极体系测 试交互反应体系中的活性铁物种的氧化还原电位. 将活化后的 S12 菌体接种于含有石墨钻电极的灭菌 反应槽 $(75 \mathrm{~mL})$ 中, 反应槽内含有 $2 \mathrm{~g} / \mathrm{L}$ 葡萄糖; 2.5 $\mathrm{g} / \mathrm{L} \mathrm{NaHCO} ; ; 0.678 \mathrm{~g} / \mathrm{L} \mathrm{NaH}_{2} \mathrm{PO}_{4} ; 0.25 \mathrm{~g} / \mathrm{L} \mathrm{NH}_{4} \mathrm{Cl} ; 0.1$ $\mathrm{g} / \mathrm{L} \mathrm{KCl} ; 0.1 \mathrm{~mol} / \mathrm{L} \mathrm{PBS}(\mathrm{pH} 7.0)$; 维生素储液 $(10 \mathrm{~mL})$; 微量元素储液 $(10 \mathrm{~mL})$. 经过 $24 \mathrm{~h}$, 菌体在石墨玷电 极形成生物膜后, 倒掉溶液. 往反应槽内加入含 $\alpha-\mathrm{FeOOH} 90 \mathrm{mmol} / \mathrm{L}$ 和乳酸钠 $10 \mathrm{mmol} / \mathrm{L}$ 的 PBS 溶 液 $(0.1 \mathrm{~mol} / \mathrm{L}, \mathrm{pH} 7.0)$ 共 $75 \mathrm{~mL}$. 以此石墨玷电极为工 作电极, 灭菌后的饱和甘永电极 (SCE)为参比电极,
另外一块石墨毡电极作为对电极放在另外一个反应 槽里面, 两块石墨玷电极大小相同 $(4.5 \mathrm{~cm} \times 4.5 \mathrm{~cm})$, 两个反应槽用阳离子交换膜连接.

\section{2 结果与讨论}

\section{1 有机氯脱氯转化动力学与氯离子生成}

图 1 显示, 3 个体系中三氯甲烷与五氯酚的还原 转化速率为 $\mathrm{S} 12+\alpha-\mathrm{FeOOH}>\alpha-\mathrm{FeOOH}>\mathrm{S} 12$. S12 对 照体系中还原转化速率很小, 三氯甲烷和五氯酚的 一级转化动力学常数 $k$ 分别为 $0.0011 \mathrm{~h}^{-1}(R=0.895)$ 和 $0.0007 \mathrm{~h}^{-1}(R=0.968) ; \alpha-\mathrm{FeOOH}$ 非生物对照体系 具有一定的有机氯转化效果, 三氯甲烷和五氯酚的 一级转化动力学常数 $k$ 分别为 $0.0058 \mathrm{~h}^{-1}(R=0.985)$ 和 $0.0045 \mathrm{~h}^{-1}(R=0.883) ; \mathrm{S} 12+\alpha-\mathrm{FeOOH}$ 交互反应体 系中有机氯转化速率明显高于两个对照体系, 三氯 甲烷和五氯酚的一级转化动力学常数 $k$ 值分别为 $0.0135 \mathrm{~h}^{-1}(R=0.989)$ 和 $0.0094 \mathrm{~h}^{-1}(R=0.978)$.

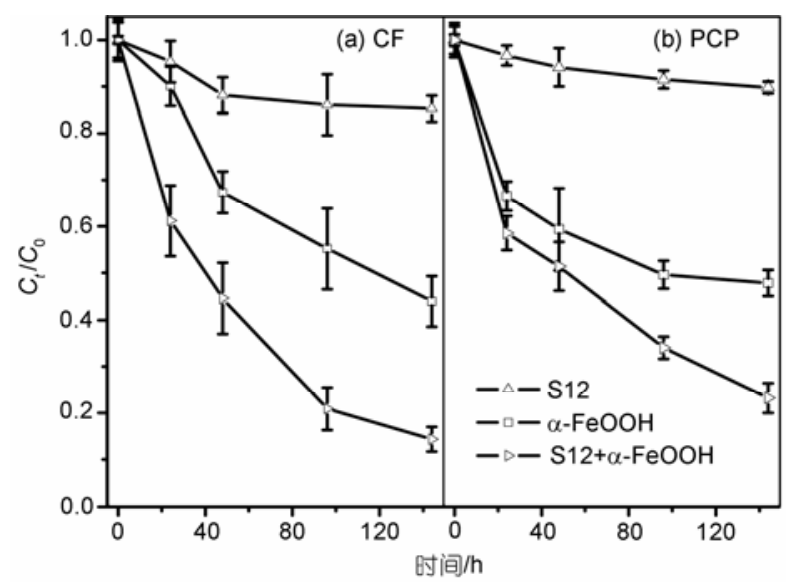

图 1 有机氯脱氯转化动力学

(a) $\mathrm{CF}$ (三氯甲烷); (b) PCP(五氯酚)

三氯甲烷和五氯酚的脱氯转化过程中氯离子的 浓度变化如图 2 所示. 为了排除反应体系所带来的氯 离子干扰，必须扣除菌体、目标物、缓冲体系等带进 反应体系的氯离子背景值和针铁矿还原溶解所产生 的氯离子背景值. 与有机氯脱氯转化动力学类似, 3 个体系氯离子产生量随时间变化规律为 $\mathrm{S} 12+\alpha-$ $\mathrm{FeOOH}>\alpha-\mathrm{FeOOH}>\mathrm{S} 12$. S12 对照体系只能检测到 微量的氯离子 $(<0.01 \mathrm{mmol} / \mathrm{L}) ; \mathrm{S} 12+\alpha-\mathrm{FeOOH}$ 交互反 应体系的氯离子浓度是 $\alpha-\mathrm{FeOOH}$ 对照体系的 2 倍以 上. 随着反应时间的增加, $\alpha-\mathrm{FeOOH}$ 对照体系与 $\mathrm{S} 12+\alpha-\mathrm{FeOOH}$ 交互反应体系中氯离子浓度均呈上升 
趋势; 而 $\mathrm{S} 12$ 对照体系中氯离子浓度没有明显变化. 反应 $144 \mathrm{~h}$ 时, $\mathrm{S} 12, \alpha-\mathrm{FeOOH}, \mathrm{S} 12+\alpha-\mathrm{FeOOH} 3$ 个体 系中三氯甲烷的脱氯率分别为 $4.2 \%, 12.0 \%, 29.3 \%$; 而五氯酚的脱氯率分别为 $2.3 \%, 12.4 \%, 24.8 \%$. 已有 一些文献证明, 某些铁还原菌能够通过胞外呼吸作 用直接以某些有机氯作为电子受体, 有机氯接受电 子而直接还原脱氯 ${ }^{[8]}$. 例如, Picardal等 ${ }^{[111}$ 证实了厌 氧条件下，腐败希瓦氏菌 200 可以通过呼吸链上的细 胞色素c催化四氯化碳还原成三氯甲烷. 从图 1 和图 2 的结果可以推断, S12 对三氯甲烷和五氯酚的直接脱 氯效果微弱, 但是 $\alpha-\mathrm{FeOOH}$ 与 $\mathrm{S} 12$ 相互作用能显著 提高还原脱氯速率.

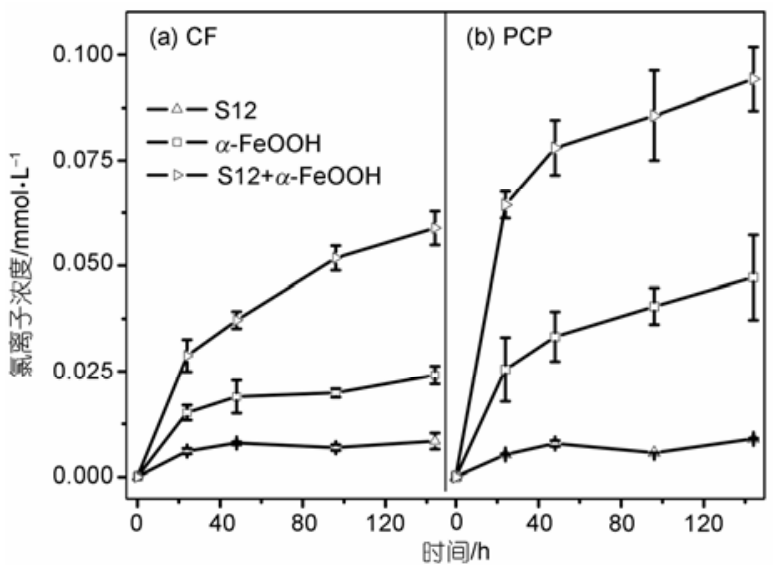

图 2 有机氯脱氯转化过程中氯离子的生成动力学 (a) $\mathrm{CF}$ (三氯甲烷); (b) PCP(五氯酚)

\section{2 吸附态 $\mathrm{Fe}(\mathrm{II})$ 生成与电化学表征}

$\mathrm{S} 12+\alpha-\mathrm{FeOOH}$ 交互反应体系具有较高的还原脱 氯能力, 可以从吸附态 $\mathrm{Fe}(\mathrm{II})$ 的大量生成及其氧化还 原电位的降低两个方面加以解释.

图 3 显示铁氧化物异化还原溶解过程中吸附态 $\mathrm{Fe}(\mathrm{II} / \mathrm{III})$ 的浓度变化. $\alpha-\mathrm{FeOOH}$ 对照体系中, 针铁矿 能够被有机酸还原溶解产生有一定量的吸附态 $\mathrm{Fe}(\mathrm{II})(<1.5 \mathrm{mmol} / \mathrm{L})^{[7]} ; \mathrm{S} 12+\alpha-\mathrm{FeOOH}$ 交互反应体系 中吸附态 $\mathrm{Fe}$ (II) 与 $\mathrm{Fe}(\mathrm{III})$ 的浓度显著高于两个对照体 系. 随着反应时间的增加, $\mathrm{S} 12+\alpha-\mathrm{FeOOH}$ 体系中吸附 态 $\mathrm{Fe}(\mathrm{II})$ 浓度呈现持续上升趋势, 这说明 $\mathrm{S} 12$ 能有效 促进 $\alpha-\mathrm{FeOOH}$ 的还原溶解生成大量的Fe( II ); 而 $\alpha-\mathrm{FeOOH}$ 非生物体系中吸附态 $\mathrm{Fe}$ ( II)呈先升后降的 趋势, 这可能因为反应过程中吸附态 $\mathrm{Fe}(\mathrm{II})$ 作用于三 氯甲烷和五氯酚的还原脱氯而被消耗掉.
采用循环伏安方法测定反应体系中活性铁物种的 氧化还原电位，如图 4 所示. S12 对照体系、 $\alpha-\mathrm{FeOOH}$ 非生物体系以及两者混合的起始点, 其 CV 图如图 4 中 $0 \mathrm{~h}$ 所表示的线; $\mathrm{S} 12$ 对照体系、 $\alpha-\mathrm{FeOOH}$ 非生物 体系在 $144 \mathrm{~h}$ 内其 $\mathrm{CV}$ 图无明显变化. 而 $\mathrm{S} 12+\alpha-$ $\mathrm{FeOOH}$ 交互反应体系, 经过 $24 \mathrm{~h}$ 反应后发现, 在 $0.104 \mathrm{~V}$ (标准电位为 $0.345 \mathrm{~V}$ ) 和 $-0.346 \mathrm{~V}$ (标准电位为 $-0.105 \mathrm{~V})$ 处出现了一对新的氧化还原峰, 此对峰是 由 $\mathrm{Fe}(\mathrm{III}) / \mathrm{Fe}$ ( II )活性铁物种引起的; 随着反应时间的 推移, 此对峰的峰强增大, 峰的位置不断负移; $144 \mathrm{~h}$ 时其氧化峰偏移至 $0.068 \mathrm{~V}$ (标准电位为 $0.309 \mathrm{~V}$ ), 还 原峰偏移至 $-0.491 \mathrm{~V}$ (标准电位为 $0.25 \mathrm{~V}$ ). 这表明 $\mathrm{S} 12+\alpha-\mathrm{FeOOH}$ 交互反应体系中, 吸附态 $\mathrm{Fe}(\mathrm{II})$ 的数

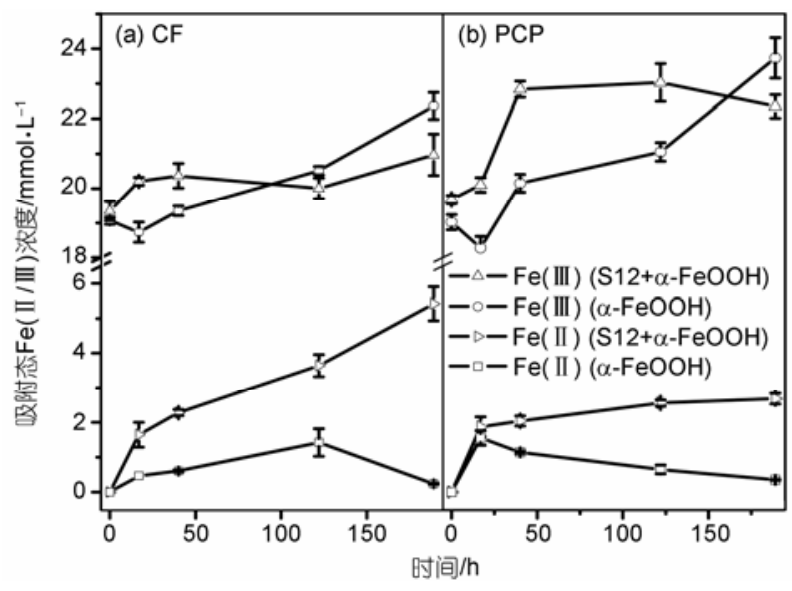

图 3 铁氧化物异化还原溶解过程中吸附态 $\mathrm{Fe}$ (II /II)的 生成动力学

(a) $\mathrm{CF}$ (三氯甲烷); (b) PCP(五氯酚)

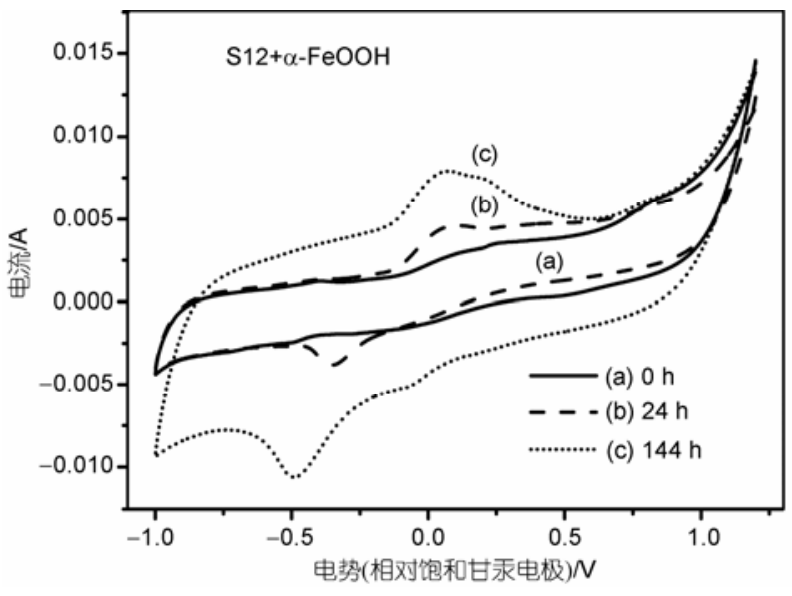

图 $4 \mathrm{~S} 12+\alpha-\mathrm{FeOOH}$ 体系中电极随时间变化的 CV 图 扫描速度为 $20 \mathrm{mV} / \mathrm{s}$ 
量不断增多, 还原能力越来越强. 由此可见, S12 还 原 $\alpha-\mathrm{FeOOH}$ 生成的吸附态 $\mathrm{Fe}(\mathrm{II})$ 具有较强的还原能 力, 这是 $\mathrm{S} 12+\alpha-\mathrm{FeOOH}$ 交互反应体系具有较高有机 氯还原脱氯效率的主要原因.

\section{3 交互反应机制探讨}

McCormick等 ${ }^{[12]}$ 报道, 还原环境下可以通过微 生物介导的生物途径和矿物介导的非生物途径来促 进四氯化碳的还原脱氯. 一方面, 某些铁还原菌可以 将有机氯作为末端电子受体，直接使其还原脱氯 ${ }^{[111}$; 另一方面, 微生物铁呼吸产生的吸附态 $\mathrm{Fe}$ (II) 可以还 原有机氯 ${ }^{[13]}$. 吸附于铁氧化物表面的 $\mathrm{Fe}(\mathrm{II})$ 能够通 过非生物途径还原有机氯, 这是因为吸附态 $F e(I I)$ 中 心周围的电子云密度增加, 提高了脱氯转化的自发 性 ${ }^{[13]}$; 而且吸附态 $\mathrm{Fe}(\mathrm{II})$ 的氧化还原电位明显低于 溶解态 $\mathrm{Fe}(\mathrm{II})$ 的 ${ }^{[14,15]}$. 图 4 的结果为这一推论提供了 直接的实验证据.

因此, 脱色希瓦氏菌 $\mathrm{S} 12+\alpha-\mathrm{FeOOH}$ 界面交互 反应体系中, 三氯甲烷和五氯酚还原脱氯转化的主 要活性物种是具有脱氯活性的吸附态 $\mathrm{Fe}$ (II), S12 则 作为有机氯脱氯转化的驱动力. 其三者之间的交互 反应机制如图 5 所示. 首先, 铁还原菌通过其特征酶 的氧化电子供体; 氧化过程中产生的电子通过呼吸 链传递给 $\alpha-\mathrm{FeOOH}$ 表面的 $\mathrm{Fe}(\mathrm{III})$, 使其还原成吸附 态 $\mathrm{Fe}(\mathrm{II})$, 这是一个生物转化过程. 然后, 吸附态 $\mathrm{Fe}(\mathrm{II})$ 与有机氯反应, 吸附态 $\mathrm{Fe}(\mathrm{II})$ 失去电子, 自身
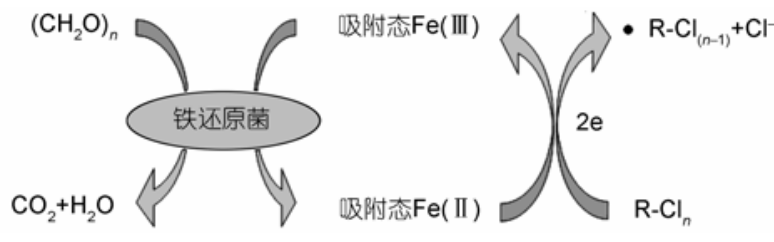

图 5 有机氯脱氯转化的铁还原菌-铁氧化物界面交互 反应机制示意图

被氧化成 $\mathrm{Fe}$ (III); 而有机氯则获得电子, 并脱下一个 氯离子, 这是一个化学转化过程. 由此, 铁氧化物界 面发生了铁还原菌介导的异化还原溶解, 并使得有 机氯发生了铁物种介导的化学脱氯反应, 这就形成 了生物与非生物的交互反应过程.

\section{3 结论}

脱色希瓦氏菌 $\mathrm{S} 12+\alpha-\mathrm{FeOOH}$ 界面交互反应体 系中, 三氯甲烷和五氯酚的还原脱氯转化速率明显 高于 $\mathrm{S} 12, \alpha-\mathrm{FeOOH}$ 对照体系. 交互反应体系具有较 高的还原脱氮能力的主要原因是 $\mathrm{S} 12$ 与 $\alpha-\mathrm{FeOOH}$ 相 互作用, 生成大量的吸附态 $\mathrm{Fe}$ (II), 并促进铁物种的 氧化还原电位持续降低, 还原活性持续提高. 这一体 系可为深入揭示自然环境中可还原性毒害物的脱毒 转化过程研究提供理论依据, 也可为可还原性毒害 物污染环境的修复与治理技术的开发提供依据. 今 后应进一步加强铁还原菌特定活性酶系、交互反应体 系电子转移途径等方面的研究.

\section{参考文献}

$1 \mathrm{Gao} \mathrm{H} \mathrm{J,} \mathrm{Jiang} \mathrm{X,} \mathrm{Wang} \mathrm{F,} \mathrm{et} \mathrm{al.} \mathrm{Residual} \mathrm{levels} \mathrm{and} \mathrm{bioaccumulation} \mathrm{of} \mathrm{chlorinated} \mathrm{persistent} \mathrm{organic} \mathrm{pollutants} \mathrm{(PO} \mathrm{Ps)} \mathrm{in} \mathrm{vegetables}$ from suburb of Nanjing, People's Republic of China. Bull Environ Contam Toxicol, 2005, 74: 673-680[D OI]

2 Wong $\mathrm{M} \mathrm{H}$, Leung A O W, Chan J K Y, et al. A review on the usage of POP pesticides in China, with emphasis on DDT loadings in human milk. Chemosphere, 2005, 60: 740-752[D OI]

3 Feng J, Lim T. Pathways and kinetics of carbon tetrachloride and chloroform reductions by nano-scale Fe and Fe/Ni particles: Comparison with commercial micro-scale Fe and Zn. Chemosphere, 2005, 59: 1267-1277ㅁD O ]

4 Tai C, Jiang G B. Dechlorination and destruction of 2,4,6-trichlorophenol and pentachlorophenol using hydrogen peroxide as the oxidant catalyzed by molybdate ions under basic condition. Chemosphere, 2005, 59: 321-326[D O I]

5 Wang $L$ G, Z hao Z H, Jiang X, et al. A ssessment of pesticide residues in two arable soils from the semi-arid and subtropical regions of China. Environ Monit A ssess, 2005, 109: 317-328[D OI]

6 Li F B, Wang X G, Liu C S, et al. Reductive transformation of pentachlorophenol on the interface of subtropical soil colloids and wa ter. Geoderma, 2008, 148: 70-78[D O I]

7 Li F B, Wang X G, Li Y T, et al. Enhancement of the reductive transformation of pentachlorophenol by polycarboxylic acids at the iron oxide-water interface. J Colloid Interface Sci, 2008, 321: 332-341[D O I]

8 Lovley D R, Holmes D E, N evin K P. Dissimilatory Fe( III) and Mn(IV) reduction. Adv Microb Physiol, 2004, 49: 219-286[D OI]

$9 \mathrm{Xu} \mathrm{M} \mathrm{Y,} \mathrm{Guo} \mathrm{J,} \mathrm{Cen} \mathrm{Y} \mathrm{H,} \mathrm{et} \mathrm{al.} \mathrm{Shewanella} \mathrm{decolorationis} \mathrm{sp.} \mathrm{nov.,} \mathrm{a} \mathrm{dye} \mathrm{decolorizing} \mathrm{bacterium} \mathrm{isolated} \mathrm{from} \mathrm{activated} \mathrm{sludge} \mathrm{of} \mathrm{a}$ 
waste-water treatment plant. Int J Syst Evol Microbiol, 2005, 55: 363-368[D O I]

10 Li X M, Zhou S G, Li F B, et al. Fe( III ) oxide reduction and carbon tetrachloride dechlorination by a newly isolated K/ebsiel/a pneumoniae strain L17. J A ppl Microbiol, 2009, 106: 130-139[D O ]

11 Picardal F W, Arnold R G, Couch H, et al. Involvement of cytochromes in the anaerobic biotransformation of tetrachloromethane by Shewanella putrefaciens 200. A ppl Environ Microbiol, 1993, 59: 3763-3770

12 McCormick M L, Bouwer E J, Adriaens P. Carbon tetrachloride transformation in a model iron-reducing culture: relative kinetics of biotic and abiotic reactions. Environ Sci T echnol, 2002, 36: $403-410$ [D O ] ]

13 A monette J E, Workman D J, Kennedy D W, et al. Dechlorination of carbon tetrachloride by Fe( II ) associated with goethite. Environ Sci Technol, 2000, 34: 4606-4613[D OI]

14 Stumm W. Chemistry of the Solid-water Interface: Processes at the Mineral-water and Particle-water Interface in Natural Systems. New York: John Wiley \& Sons, 1992

15 Z egeye A, Ruby C, Jorand F. Kinetic and thermodynamic analysis during dissimilatory $\gamma$ - $\mathrm{FeO} \mathrm{OH}$ reduction: Formation of green rust 1 and magnetite. Geomicrobiol J, 2007, 24: 51-64[D O I]

动 态.

\section{量子计算第一步：合成和控制预定的量子态}

量子力学在过去的一个世纪获得了长足的发展. 其 中许多反直觉的预言(如不确定性原理、叠加态和纠缠态) 已经在物理学的各个分支领域被实验所证实. 近年来, 人 们认识到有些看似荒谬的理论在许多领域也有其应用价 值, 如: 量子密码术和量子计算等. 这一认识激发了科学 家对于“量子态加工技术”的兴趣, 这些技术很可能在未来 的量子计算中发挥作用. 一般认为, 量子力学主要对原 子、原子核和电子等微观系统起作用, 但最近的研究表明, 在人工设计的超导线路(微米或毫米尺寸)中, 量子力学行 为也能得到控制. 由超导电感和电容组成的线性谐振器是 最简单的超导量子线路, 它可以接受一个一个的(微波频 率)光子注入并存储这些光子. 另外一种超导量子线路是 使用约瑟夫森(Josephson)结(作为非线性电感)构成的量子 比特(qubit), Josephson qubit 是一个双态系统, 具有基态 $|g\rangle$ 和激发态 $|\mathrm{e}\rangle$.

最近, 来自美国加州大学(Santa Barbara)的 Max Hofheinz 等人将上述超导谐振器与超导量子比特耦合, 在 谐振器中制备出了事先设计好的量子态. Josephson qubit 在耦合中起一个“光子铲车”的作用, 将光子一个接一个地 装入谐振器. 研究者使用新构建的装置, 在装载每一个光 子的过程中, 不仅控制 qubit 中叠加态的幅值, 也控制
基态 $|\mathrm{g}\rangle$ 和激发态 $|\mathrm{e}\rangle$ 的相对位相, 如精确装载波函数为 $(1 / \sqrt{ } 2)(|\mathrm{g}\rangle-\mathrm{i}|\mathrm{e}\rangle)$ 的叠加态. 最终, 在谐振器中得到了多种 复合 $\mathrm{N}$-光子态, 它们分别满足事先对终态的设计. Hofheinz 等人应用 Wigner 层析法使各种终态成为可见. 在医学 诊断中使用的“X 射线-CT” 或“核磁共振”, 可以采用 Wigner 层析法通过不同视角的大量测量(使用全同的 qubit 作为终态诊断的探针, 卸载谐振器的 N-光子态)重构量子 态的图像.

这项研究最值得注意的进展在于：在谐振器中产生 的目标终态, 完全是预先设计好的, 研究者将其定量表示 为 Wigner 函数. 在研究报告中, 作者分别展示了根据 Wigner 函数作出的计算机模拟图像和根据实测得到的 Wigner层析图像. 两种图像看上去几乎没有差别. 这意味 着所合成的目标终态具有高保真度, 从而满足了实现量 子计算的先决条件. 此外, 作者在此次研究中所实施的量 子比特与谐振器之间的耦合, 也为末来量子计算中的逻 辑门操作提供了技术基础. 目前尚存的问题是在超导线 路中的量子退相干问题. 在接下来的工作中, 需要改进线 路设计, 减少线路材料中的缺陷数, 进一步弄懂退相干的 机制.

(戴闻 编译自 Nature, 2009, (459): 516, 546) 